\title{
Patterns of wrist cutting: A retrospective analysis of 115 suicide attempts
}

\author{
Jakwang Cho, Youngwoong Choi \\ Department of Plastic and Reconstructive Surgery, Inje University Sanggye Paik Hospital, Inje University College of Medicine, Seoul, Korea
}

Background Rosenthal et al. classified female, habitual, non-suicidal wrist cutters as a group and introduced the concept of wrist-cutting syndrome. We investigated the characteristics of wrist-cutting patients at our institution in comparison with results reported previously. Methods We conducted a retrospective study involving 115 patients who had cut their wrists and been examined at the emergency department of a single hospital in Seoul, Korea, between March 2014 and August 2018.

Results There were more women (73 patients; 63.5\%) than men (42 patients; 36.5\%), and the women (mean age, 34.42 years) were significantly younger than the men (mean age, 50.07 years). The patients who had cut their wrists repeatedly were mainly women (22 of 26 patients; 84.6\%); however, men caused more severe damage than women. Substance use before a suicide attempt did not significantly increase the severity of wrist cutting. Our institution planned and implemented a suicide prevention intervention program to improve the continuity of outpatient care. The number of patients who continued psychiatric treatment increased significantly after program completion.

Conclusions We confirmed that most patients were young women who were not suicidal in the true sense because their wounds were not severe. Our study showed a protective role of the barrier tendons (flexor carpi radialis, palmaris longus, flexor carpi ulnaris), and we suggest careful repair of the barrier tendons to protect neurovascular structures against subsequent cutting events. We found that it was possible to improve the continuity of patient counseling by managing patients through a psychiatric treatment program.

Keywords Attempted suicide / Tendon injury / Psychology / Barrier / Wrist injuries

Received: January 17, $2020 \bullet$ Revised: April 29, $2020 \bullet$ Accepted: May 5, 2020

pISSN: 2234-6163 • elSSN: 2234-6171 • https://doi.org/10.5999/aps.2020.00059・Arch Plast Surg 2020;47:250-255
Correspondence: Youngwoong Choi Department of Plastic and

Reconstructive Surgery, Inje University Sanggye Paik Hospital, Inje University College of Medicine, 1342 Dongil-ro, Nowon-gu, Seoul 01757, Korea

Tel: +82-2-950-1048

Fax: +82-2-932-6373

E-mail:pshero2@naver.com

\section{INTRODUCTION}

Wrist cutting has been a medical concern for Western psychiatrists and psychologists since the 1960s [1]. Since then, epidemiological studies have analyzed the psychological reasons for and aspects of self-inflicted wrist cutting through clinical evaluations of patients. The concept was introduced to Japan in the 1970s, when Rosenthal et al. proposed the concept of wrist-cut- ting syndrome, leading to a few related epidemiological studies in Japan [1-3]. According to these studies, most patients were women, and their injuries were non-suicidal and minor. This pattern of self-injurious behavior was shown to be without suicidal intention and used as a tool for relieving psychological tension. Moreover, Simpson [3] reported that wrist cutters frequently experienced unpredictable mood swings and depersonalization, while after self-cutting, they felt relaxation and reper- 
sonalization, which prompted him to term this behavior "antisuicide."

In this study, we examined the epidemiology, severity, psychological features, and anatomical features of cases of self-inflicted wrist-cutting and explored correlations between these factors. It has been decades since the concepts of "wrist-cutting syndrome" and "anti-suicide" were first introduced, and we investigated whether these frameworks are still valid. In addition, active research on wrist cutting has been conducted in Japan, but there have been no similar studies in Korea or any other Asian country [1].

\section{METHODS}

\section{Patient selection}

We conducted a retrospective study of 115 patients who had cut their wrists and were examined at the emergency department by members of the department of plastic and reconstructive surgery at a single hospital in Seoul, Korea, between March 2014 and August 2018. This study was approved by the Institutional Review Board (IRB No. 2020-02-013). The inclusion criterion was a wrist-cutting event, defined as a skin lesion present on the wrist ( $10 \mathrm{~cm}$ proximal to the distal wrist crease) with the intention of self-harm.

\section{Methods}

The first evaluation was performed at the emergency department, and all patients were analyzed for alcohol consumption, drug abuse, psychological state, and previous suicide attempts. The clinical features of patients' wounds, including the injury side, the injury pattern, and the tool used, were recorded, and damage to anatomical structures was recorded as part of the initial evaluation. These data were retained in computerized records, which contained each patient's sex, age, and the clinical features of the injury, and the data were processed for anonymous use in this study. All patients provided informed verbal consent, and we anonymized our data.

Alcohol intake was defined as the consumption of at least one drink of alcohol during the 24 hours preceding the wrist-cutting incident. This variable was recorded through history-taking, along with laboratory tests. Drug intoxication was defined as the consumption of any drug before wrist cutting. Known psychiatric diseases were classified according to the diagnostic criteria in the diagnostic and statistical manual of mental disorders, fifth edition [4]. Patients who met the criteria for two or more known psychiatric diseases were counted in each category.

For patients who had cut their wrists multiple times and had been treated at the emergency department twice or more, only the last event was analyzed in this study, while earlier events were recorded as previous suicide attempts. All patients were advised to seek psychiatric treatment before injury evaluation and surgical treatment, and psychiatric treatment was provided only with patients' consent. For those who consented, a psychiatric consultation and diagnosis were performed in the emergency room, after which outpatient care was recommended. If necessary, patients were hospitalized in a closed or general ward. As few patients had regularly received outpatient care, our institute planned and implemented a suicide prevention intervention (SPI) program starting on March 13, 2017. In the SPI program, we contacted and supported patients who had not received regular outpatient care. In addition, we attempted to persuade patients to visit the hospital to receive appropriate treatment.

\section{Schematization of the wrist structure}

Severity was classified according to the depth-dependent damage of the anatomical structure. In several previous studies examining wrist injury patterns, the three-layered taxonomy introduced by Lee et al. was used to distinguish the severity of wrist injuries $[5,6]$. These studies described the different patterns of damage and anatomical features of self-inflicted wrist injuries and wrist injuries caused by other reasons without suicidal intention (e.g., accidental damage without intention or stab and penetrating wounds of the wrist during a fight or violent event involving another person). We noted that deep layer injuries, the worst in the three-layered taxonomy, were significantly less common in self-cutting patients than in the other two groups.

Therefore, in the current study, we classified anatomical injuries dichotomously. We created a virtual line on the anatomy of the wrist, immediately above the median nerve, to examine the relationship between various patient-related factors and the severity of the wrist-cutting injury. If structures beneath this virtual line were damaged, the injury was classified as deep, while injuries to anatomical structures located above this virtual line were classified as superficial. The rationale for this classification was that injury of neurovascular structures, including the ulnar artery and radial artery, can lead to critical self-harm. Skin-only injuries with subcutaneous fat exposure were considered to be the most minor. We simplified the anatomy of the wrist structures in a schematic manner and the relative location of each structure was drawn according to the study by Lee et al. [5], structures 1-7 were defined as the superficial layer. These structures correspond to the palmaris longus (PL), flexor carpi radialis (FCR), second flexor digitorum superficialis (2-FDS), 3-FDS, 4-FDS, 5-FDS, and flexor carpi ulnaris (FCU), respectively. The deep layer comprised structures 8-16, which correspond to the radial 


\section{Fig. 1. Schematic representation of 16 wrist structures}

Injuries were dichotomized as deep or superficial based on their location relative to a virtual line running immediately above the median nerve. In accordance with the study by Lee et al. [5], structures 1-7 were defined as the superficial layer. These structures correspond to the palmaris longus, flexor carpi radialis, second flexor digitorum superficialis (2-FDS), 3-FDS, 4-FDS, 5-FDS, and flexor carpi ulnaris, respectively. The deep layer comprised structures 8-16, which correspond to the radial artery, flexor pollicis longus, median nerve, second flexor digitorum profundus (2-FDP), 3-FDP, 4-FDP, 5 -FDP, ulnar nerve, and ulnar artery, respectively.

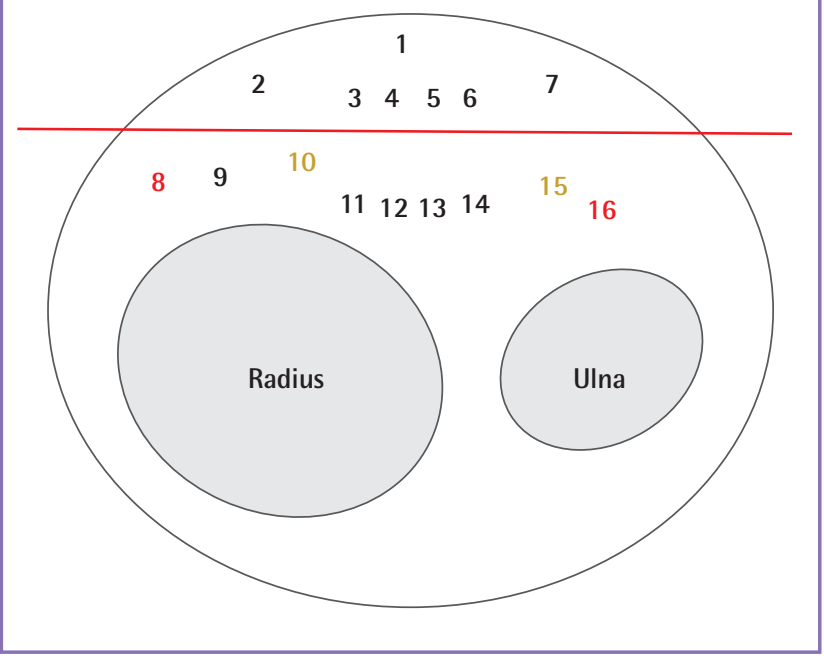

artery, flexor pollicis longus (FPL), median nerve, second flexor digitorum profundus (2-FDP), 3-FDP, 4-FDP, 5-FDP, ulnar nerve, and ulnar artery, respectively (Fig. 1).

\section{Statistical analysis}

The demographic data were analyzed with the significance level set at $\mathrm{P}<0.05$. As we categorized the severity of wrist injuries according to three levels (skin only, superficial, and deep), the relationships of various factors with severity was evaluated using the linear-by-linear association test. The sex ratio was compared to the cumulative population of Seoul during the study period (2014-2018) [7]. The sex ratios for incidence and repeated wrist cutting were evaluated using the chi-square test. The mean ages of men and women were compared using the independent t-test.

\section{RESULTS}

\section{Patient epidemiology}

This study included 115 patients who had cut their wrists for the purpose of self-harm, of whom 42 patients (36.5\%) and 73 patients $(63.5 \%)$ were men and women, respectively (Table 1 ). The ratio of women to men was significantly higher $(\mathrm{P}<0.05)$ in this study than in the cumulative population of Seoul during
Table 1. Demographic and clinical features of the patients

\begin{tabular}{|lc|}
\hline Variable & No. of patients (\%) \\
\hline Total number & 115 \\
Sex & \\
Male & $42(36.5)$ \\
Female & $73(63.5)$ \\
Age, mean \pm SD (range), yr & $18.91(15-86)$ \\
Side & \\
Left & $101(87.8)$ \\
Right & $7(6.1)$ \\
Both & $7(6.1)$ \\
Aspect & \\
Volar & $108(93.9)$ \\
Dorsal & $1(0.9)$ \\
Volar and dorsal & $5(4.3)$ \\
Amputation & $1(0.9)$ \\
Depth & \\
Skin only & $82(71.3)$ \\
Superficial & $20(17.4)$ \\
Deep & $13(11.3)$ \\
\hline
\end{tabular}

the study period (2014-2018) [7].

The patients' mean age was 40.14 years. Women were significantly younger than men (mean age, 34.42 and 50.07 years, respectively; $\mathrm{P}<0.05$ ). Alcohol consumption and drug abuse prior to wrist cutting were examined in all patients. Fifty-one patients (44.3\%) had consumed alcohol before cutting their wrists, 11 (9.6\%) had consumed drugs, and five (4.3\%) had done both. In cases involving drug intoxication, the most common drugs used were prescription pills (e.g., sleeping pills, mood stabilizers, and antidepressants). In one case, a patient consumed a pesticide ( $50 \mathrm{~mL}$ of cypermethrin) that was prepared in advance with the intention of committing suicide. However, substance abuse did not significantly increase the severity of wrist cutting (alcohol consumption: $\mathrm{P}=0.752$, drug abuse: $\mathrm{P}=0.853$ ).

\section{Psychiatric features}

Of the 115 patients, 47 (40.9\%) had a known psychiatric disease, and $36(76.6 \%)$ had been diagnosed with a mood disorder (Table 2). In total, 47 patients (40.9\%) consented to undergo a psychiatric consultation and treatment in the emergency department, of whom 31 (27.0\%) were treated in the outpatient department or ward during the study period. For patients who declined psychological counseling, we made several recommendations that they receive psychiatric care through the SPI. After the SPI, there was a significant increase in the number of patients who continued psychiatric treatment $(\mathrm{P}<0.05)$. The most adherent patient completed 10 SPI sessions.

Twenty-six patients (22.6\%) had attempted suicide by wrist 
Table 2. Known psychiatric history of patients who performed wrist-cutting

\begin{tabular}{|lc|}
\hline Psychiatric history & No. of patients (\%) \\
\hline Mood disorders & $36(76.6)$ \\
Schizophrenia spectrum and other psychotic disorders & $4(8.5)$ \\
Substance-related disorders & $6(12.8)$ \\
Anxiety disorders & $7(14.9)$ \\
Impulse control disorders & $5(10.6)$ \\
Sleep-wake disorders & $2(4.3)$ \\
Neurocognitive disorders & $2(4.3)$ \\
\hline
\end{tabular}

cutting on previous occasions, of whom 16 had engaged only in wrist cutting, while two had additionally attempted hanging, two had overdosed on drugs, and six had overdosed on drugs and then engaged in self-harm. Of the 22 patients who had engaged in wrist cutting repeatedly, 19 (86.4\%) were women and three $(13.6 \%)$ were men, demonstrating a significant predominance of women in cases involving previous wrist cutting $(\mathrm{P}<0.05)$.

\section{Site of injury}

The vast majority of patients (101 patients; $87.8 \%$ ) had cut only the left wrist, while seven $(6.1 \%)$ had cut only the right wrist and seven $(6.1 \%)$ had cut both wrists. Among the 101 patients with a left-side wound only, 92 (92\%) had a right-side dominant hand. In addition, 108 patients (93.9\%) had cut only the volar side, one $(0.9 \%)$ had cut only the dorsal side, and five $(4.3 \%)$ had cut both the volar and dorsal sides. One patient performed amputation after cutting her wrist for 3 hours.

\section{Severity}

In total, 82 patients (71.3\%) had skin-only injuries, without any injury to anatomical structures of the wrist, 20 (17.4\%) had superficial injuries, and 13 (11.3\%) had deep injuries. None of the patients died.

Thirty-three patients had injured at least one anatomical structure of the wrist. The number of patients who injured each anatomical structure of the wrist are listed in Table 3. The patient who performed amputation was counted once in all cases. The most commonly injured structure was the PL, which was injured in 24 patients (72.7\%). The most commonly injured structure in the deep layer was the median nerve, which was injured in 10 patients (30.3\%). Previous studies have pointed out the protective role of some tendons against neurovascular injuries $[5,6]$. Specifically, those studies indicated that the FCR functions as an important protective barrier for the radial artery, and the PL exhibits a similar effect for the median nerve. In our study, all five patients with radial artery injuries had FCR inju-
Table 3. The number of patients who injured each anatomical structure $(n=33)$

\begin{tabular}{|lc|}
\hline Variable & No. of patients (\%) \\
\hline Palmaris longus & $24(72.7)$ \\
Flexor carpi radialis & $15(45.5)$ \\
Flexor carpi ulnaris & $13(39.4)$ \\
Flexor digitorum superficialis & $12(36.4)$ \\
Flexor digitorum profundus & $2(6.1)$ \\
Flexor pollicis longus & $2(6.1)$ \\
Median nerve & $10(30.3)$ \\
Ulnar nerve & $1(3.0)$ \\
Radial artery & $5(15.2)$ \\
Ulnar artery & $2(6.1)$ \\
\hline
\end{tabular}

ries and all 10 patients with median nerve injuries had PL injuries; therefore, our findings validate the proposal that tendons are protective structures.

Sex influenced the severity of self-inflicted wrist cutting, as men had significantly more severe injuries than women $(\mathrm{P}<$ $0.05)$.

\section{Treatment}

Of the 115 patients, three refused treatment for the wound, while 113 received skin repair. Tenorrhaphy was performed in 26 patients (23.0\%), and myorrhaphy was performed in six patients (5.3\%). Neurorrhaphy, arteriorrhaphy, and venorrhaphy were performed in 13 (11.5\%), eight (7.1\%), and three (2.7\%) patients, respectively. Fascia reconstruction and nerve sheath reconstruction were performed in two patients each, with an acellular dermal matrix used to improve the prognosis. Replantation surgery was conducted in the patient who performed amputation.

\section{DISCUSSION}

Attempted suicide by wrist cutting is a major social problem, accounting for a large proportion of cases of upper extremity trauma presenting to the emergency room. Hence, many epidemiological studies have been conducted from a psychiatric perspective to examine the clinical features of self-inflicted wrist cutting [1-3]. Our results are consistent with those of previous studies on this topic. Most patients were young women who were not suicidal in the true sense because their wounds were not severe, aligning with the concept of wrist cutting as "anti-suicide" [3]. In addition, most patients who had engaged in wrist cutting repeatedly were women (Table 1 ) $[8,9]$.

Several studies have examined the relationship of suicide with substance use, one of which showed an association between alcohol consumption and suicide [10]. However, in the current study, alcohol consumption and substance abuse did not in- 
crease the severity of the injury or the risk of mortality in wristcutting patients.

In a study examining the epidemiology of wrist cutting in psychiatric inpatients, the proportion of patients with schizophrenia (36.4\%) was similar to that of patients with mood disorders [11]. In contrast, most patients in the current study with a psychiatric history had mood disorders (36 patients; 76.6\%), and only four (8.5\%) had schizophrenia (Table 2). These findings are consistent with the interpretation that many of the patients who cut their wrists and visited the emergency department had attempted "anti-suicide" because they were in a depersonalized state resulting from depression [12].

A large number of patients wanted only surgical treatment for their injuries, and they commonly considered their psychiatric disorders minor. Since approximately $50 \%$ to $60 \%$ of people who commit suicide have a history of self-harm, those who cut their wrists require regular psychiatric counseling to prevent devastating outcomes [11]. Our institution has shown that it is possible to improve the continuity of patient counseling by managing patients through psychiatric treatment program such as our SPI. Our study is highly meaningful as the first study conducted in Korea to examine the psychological aspects of wrist cutting, and our findings indicate that repetitive wrist cutting can be prevented by ensuring regular contact with patients so that they continue to receive psychiatric consultations.

Thus, physicians treating wrist-cutting patients should be wellinformed regarding the epidemiology and psychologic features of these patients and should encourage them to receive subsequent psychiatric counseling as well as surgical treatment. In the few studies that have examined wrist injury patterns, PL and median nerve injuries were significantly over-represented in selfcutting injuries than in other wrist injuries without suicidal intention $[5,6]$. Likewise, our study showed that PL injuries were the most common anatomical structure injuries $(72.7 \%, 24 / 33)$ and that median nerve injuries were the most common anatomical structure injuries in the deep layer $(30.3 \%, 10 / 33)$. Therefore, we emphasize that surgeons should carefully examine the structures that are most likely to be damaged after detailed history-taking in wrist-cutting patients.

The barrier effect of the FCR, PL, and FCU as tendons in the superficial layer on neurovascular structures has been verified $[5,6]$. Likewise, in our study, the protective roles of the PL against median nerve injury and of the FCR against radial artery injury were confirmed. Based on these findings, surgeons should be able to predict the possibility of neurovascular injury if damage of a barrier tendon is identified in the initial exploration of the wounds. In addition, surgeons should be aware that wrist-cutting patients, in light of the concept of wrist-cutting syndrome, are likely to repeatedly injure their wrists. Therefore, we suggest careful repair of the barrier tendons (FCR, PL, and FCU) for the protection of neurovascular structures against subsequent cutting events. In comparison with previous research, a remarkable aspect of this study is that regular psychiatric consultations, as facilitated through the SPI program, might significantly reduce the recurrence of predictable wrist cutting. Therefore, physicians who treat wrist-cutting patients should reencourage them to continue psychiatric counseling in addition to performing an accurate evaluation and appropriate surgical treatment.

The study was subject to certain limitations. For example, it was conducted at a single institution and was retrospective in nature. Moreover, patients were not monitored closely, and follow-up investigations of hand function were not performed after surgery. In addition, the study period was short, and the sample size was small. Another limitation is that the subjects of this investigation were limited to patients with self-cutting wrist injuries. Unlike previous studies that compared wrist injuries with multiple causes, no comparisons were made with patients with wrist injuries caused by other pathways because this study focused only on wrist-cutting patients $[5,6]$. In the same vein, the dichotomous schematization of the wrist structures could be noted as a limitation. However, the rationale for our schematic representation, which combined the middle layer and deep layer, was that injuries to the deepest wrist structures were significantly underrepresented in wrist-cutting patients compared to patients with wrist injuries from other causes. Our results showed similar results to those reported in previous studies on the epidemiology of wrist-cutting patients. Most patients were young women who were not suicidal in the true sense because their wounds were not severe. Most patients who had cut their wrists repeatedly were women [9]. We found that it was possible to improve the efficacy of patient counseling by managing patients through a psychiatric treatment program. Finally, for hand surgeons, we suggest careful repair of the barrier tendons (FCR, PL, and FCU) to protect neurovascular structures against subsequent cutting events.

\section{NOTES}

\section{Conflict of interest}

No potential conflict of interest relevant to this article was reported.

\section{Ethical approval}

The study was approved by the Institutional Review Board of Inje University Sanggye Paik Hospital (IRB No. 2020-02-013) 
and performed in accordance with the principles of the Declaration of Helsinki.

\section{Author contribution}

Conceptualization: Y Choi. Data curation: J Cho. Formal analysis: J Cho. Methodology: Y Choi. Writing - original draft: J Cho. Writing - review \& editing: Y Choi. Approval of final manuscript: all authors.

\section{ORCID}

Jakwang Cho https://orcid.org/0000-0003-4707-2901

Youngwoong Choi https://orcid.org/0000-0002-2211-2981

\section{REFERENCES}

1. Matsumoto T, Yamaguchi A, Chiba Y, et al. Patterns of selfcutting: a preliminary study on differences in clinical implications between wrist- and arm-cutting using a Japanese juvenile detention center sample. Psychiatry Clin Neurosci 2004;58:377-82.

2. Rosenthal RJ, Rinzler C, Wallsh R, et al. Wrist-cutting syndrome: the meaning of a gesture. Am J Psychiatry 1972;128: 1363-8.

3. Simpson MA. The phenomenology of self-mutilation in a general hospital setting. Can Psychiatr Assoc J 1975;20:42934.
4. American Psychiatric Association. Diagnostic and statistical manual of mental disorders. 5th ed. Arlington: American Psychiatric Publishing; 2013.

5. Lee $\mathrm{CH}$, Cha SM, Shin HD. Injury patterns and the role of tendons in protecting neurovascular structures in wrist injuries. Injury 2016;47:1264-9.

6. Kisch T, Matzkeit N, Waldmann A, et al. The reason matters: deep wrist injury patterns differ with intentionality (accident versus suicide attempt). Plast Reconstr Surg Glob Open 2019; 7:e2139.

7. Statistics Korea. Population Census [Internet]. Daejeon: Statistics Korea [cited 2018 Oct 30]. Available from: http:// kostat.go.kr/portal/korea/index.action.

8. Chuinard RG, Friermood TG, Lipscomb PR. The 'suicide' wrist: epidemiologic study of the injury. Orthopedics 1979; 2:499-503.

9. Ersen B, Kahveci R, Saki MC, et al. Analysis of 41 suicide attempts by wrist cutting: a retrospective analysis. Eur J Trauma Emerg Surg 2017;43:129-35.

10. Razvodovsky YE. Suicide and alcohol psychoses in Belarus 1970-2005. Crisis 2007;28:61-6.

11. Hawton K, Bergen H, Cooper J, et al. Suicide following selfharm: findings from the multicentre study of self-harm in England, 2000-2012. J Affect Disord 2015;175:147-51.

12. Suyemoto KL. The functions of self-mutilation. Clin Psychol Rev 1998;18:531-54. 